\title{
Unique paths as formal points
}

\author{
THIERRY COQUAND \\ PETER SCHUSTER
}

\begin{abstract}
A point-free formulation of the König Lemma for trees with uniformly at most one infinite path allows for a constructive proof without unique choice.

2000 Mathematics Subject Classification: 03E25, 03F65 (primary); 05C05, 05C38, 05 C63 (secondary)
\end{abstract}

Keywords: König Lemma, point-free, constructive, unique choice

\section{Introduction}

The (Weak) König Lemma says that every infinite (binary) tree has an infinite path. Kleene [17] gave a recursive counterexample to this statement: a recursive infinite binary tree without recursive infinite path. Moreover, the Weak König Lemma for detachable trees (WKL) is equivalent $[13,4]$ to Bishop's Lesser Limited Principle of Omniscience (LLPO) - a fragment of the Law of Excluded Middle that fails to hold recursively-in conjunction with an instance of dependent choice. One thus cannot expect to find a constructive proof of WKL as it stands, let alone of König's Lemma.

By a constructive proof we mean one that is carried out within Bishop-style constructive mathematics (BISH) [5], the overall framework of this note. Due to the choice of intuitionistic logic characteristic of BISH, one or the other assumption needs to be made explicit that would be automatic with classical logic: an assertion $A$ is said to be decidable whenever $A \vee \neg A$ holds; ${ }^{1}$ a subset $S$ of a set $T$ is called detachable if $x \in S$ is decidable for each $x \in T$; and a set $S$ is named discrete if $x=y$ is decidable for each pair $x, y \in S$. Likewise, one says that a set $S$ is inhabited if it has an element, which is to be distinguished from the double negation that $S$ be nonempty.

After this digression on the method of the present paper, we come back to its principal object: infinite paths of infinite trees. Kleene's example can be ruled out by requiring

\footnotetext{
${ }^{1}$ For a brief discussion of this use of "decidable" in contrast to "recursive" we refer to [16]. Note in this context that every theorem of BISH is valid in Russian recursive mathematics, which is BISH plus Markov's principle and (an appropriate form of) Church's thesis [10].
} 
that each tree under consideration have at most one infinite path. When one restricts WKL to trees of this sort, the resulting principle WKL! is equivalent $[3,15,30]$ to Brouwer's Fan Theorem for detachable bars (FAN). This parallels the equivalence [2] of FAN and the minimum principle MIN! analogous to WKL! An analysis [28] of the latter equivalence has shown, however, that FAN is only needed for the step from the given uniqueness hypothesis to the canonical uniform variant of it. In fact FAN is necessary and sufficient for this first step, whereas the second step, from the uniform uniqueness precondition to the desired existence statement, is a mere instance of completeness. ${ }^{2}$

To analyse the equivalence of FAN and WKL! in a similar way, we recall $[15,30]$ that FAN suffices for the assertion (*) "every detachable binary tree with at most one infinite path has uniformly at most one infinite path". Moreover, $(*)$ implies WKL!: every detachable binary tree with uniformly at most one infinite path has a longest path [15], which is an infinite path of the tree whenever, in addition, the tree is infinite. (In fact WKL is equivalent [14] to the Longest Path Lemma (LPL): every detachable binary tree has a longest path.) In combination with the implication from WKL! to FAN, this yields that FAN is also necessary for the assertion (*).

In the present paper we give a direct proof of the statement that every infinite tree with uniformly at most one infinite path actually has an infinite path. It is noteworthy that the tree under consideration need neither be detachable nor binary; it suffices that its nodes are finite words over a discrete alphabet. As a by-product we observe that if-as in the binary case - the alphabet happens to be finite, then for every detachable binary tree with uniformly at most one infinite path there is a spread which is an infinite path of the given tree whenever the tree is infinite. The spread we construct need not be contained in the tree under consideration unless this is infinite; at least in this sense the spread is a generalisation of a longest path.

In the spirit of formal topology [26,27] we understand infinite paths as linear spreadsor, equivalently, as infinite chains-rather than as infinite sequences. This allows us

\footnotetext{
${ }^{2}$ As a proof paradigm this instance of completeness can be traced back to Russian recursive mathematics [11,23] (see also [22]; applications include the construction of Haar measures); has proved productive in constructive analysis see [7, 8] (see also [9] and [5, Chapter 7]; the use of uniform uniqueness is often somewhat implicit [12]); stood right at the beginnings of proof mining [18, 19] (see also [20] and [21, Chapters 15-7]; here uniform uniqueness is put as a modulus of uniqueness); has repeatedly shown up in computable analysis [6,32,33]; and most recently has reoccurred in abstract Stone duality [1, 31]. We refer to [29] for a reminder of this time-honoured argument, for a treatment of it in a choice-free environment, and for a few more details about its impressive history.
} 
to get by without any choice, whereas one might have to use unique countable choice to extract an infinite sequence from an infinite path. We also work with a first-order characterisation of "uniformly at most one infinite path" [15, 30], which, in particular, is formulated without infinite sequences. Spreads have further been used in contexts which lack uniqueness, to avoid choosing sequences [25].

\section{Preliminaries}

By $m, n, k, \ldots$ we mean integers $\geqslant 0$. Let $A$ throughout be an inhabited discrete set. Examples are $A=\mathbb{N}$ and $A=\{0,1\}$. We use $u, v, w, \ldots$ as variables for the elements of

$$
A^{*}=\bigcup_{n \geqslant 0} A^{n}
$$

As usual the length of $u \in A^{n}$ is defined as $|u|=n$. The empty sequence () is the only one of length 0 . The $n$-th finite initial segment

$$
\bar{u} n=(u(0), \ldots, u(n-1))
$$

of $u$ with $|u| \geqslant n$ has length $n$. Note that $\bar{u}|u|=u$ and $\bar{u} 0=()$.

We say that a subset $T$ of $A^{*}$ is unbounded (in length) if

$$
\forall n \exists u(u \in T \wedge|u| \geqslant n) \text {, }
$$

and bounded (in length) if

$$
\exists n \forall u(u \in T \rightarrow|u|<n) .
$$

If $A$ is finite, which we do not suppose in general, then $T \subseteq A^{*}$ is (un)bounded precisely when it is (in)finite.

We identify each sequence ( $i$ ) of length 1 with its only element $i \in A$, and denote the concatenation of $u$ and $v$ by their juxtaposition $u v$. For example, if $w=u i$ for some $i \in A$, then $w$ is an immediate successor of $u$. We write $u \leqslant w$ if $u$ is a restriction of $w$ : that is,

$$
u \leqslant w \leftrightarrow \exists v(u v=w) .
$$

This partial order on $A^{*}$ is decidable, because $A$ is discrete. Clearly, () is the least element of $A^{*}$ with respect to $\leqslant$.

A subset $T$ of $A^{*}$ is closed under restrictions if

$$
u \leqslant w \rightarrow w \in T \rightarrow u \in T .
$$


Any such $T$ is inhabited if and only if ()$\in T$; moreover, it is unbounded precisely when

$$
\forall n \exists u(|u|=n \wedge u \in T),
$$

and bounded precisely when

$$
\exists n \forall u(|u|=n \rightarrow u \notin T) .
$$

A tree is an inhabited subset $T$ of $A^{*}$ which is closed under restrictions. A tree $T$ is a spread if every element of $T$ has an (immediate) successor in $T$ : that is,

$$
\forall u(u \in T \rightarrow \exists i \in A(u i \in T)) .
$$

If $T$ is a spread, then-by induction-for every $n$ there is $u \in T$ with $|u|=n$, which is to say that $T$ is unbounded; in other words, a spread $T$ has at least one element of any given length. Note that we do not generally suppose that a tree be detachable.

A subset $T$ of $A^{*}$ is a chain if it is linearly ordered with respect to $\leqslant$ or, equivalently, if

$$
u, u^{\prime} \in T \rightarrow|u| \leqslant\left|u^{\prime}\right| \rightarrow u \leqslant u^{\prime} .
$$

In particular, a chain $T$ has at most one element $u$ of any given length:

$$
u, u^{\prime} \in T \rightarrow|u|=\left|u^{\prime}\right| \rightarrow u=u^{\prime} ;
$$

this condition is even equivalent to " $T$ is a chain" whenever $T$ is closed under restrictions. A chain is (un)bounded precisely when it is (in)finite. By a linear spread we understand a spread which is a chain. It is easy to see that the linear spreads are precisely the infinite chains. Clearly a linear spread has exactly one element of any given length.

\section{Infinite paths}

The following definitions will be used throughout this section. Let $T$ be a tree. We consider the predicate

$$
P(u, k) \equiv \forall w(|w|=|u|+k \rightarrow w \in T \rightarrow u \leqslant w),
$$

for which $P(u, k)$ implies $P(u, \ell)$ whenever $k \leqslant \ell$. We further set

$$
S=\left\{u \in A^{*}: \exists k P(u, k)\right\} .
$$

If $T$ is detachable, and $A$ is finite, then $P$ is decidable, and thus $S$ simply existential $\left(\Sigma_{1}^{0}\right)$. 


\section{Lemma 1}

(1) The subset $S$ of $A^{*}$ is a tree.

(2) If $T$ is bounded, then $S=A^{*}$.

(3) If $T$ is unbounded, then $S$ is a chain that satisfies $S \subseteq T$.

Proof The only perhaps not obvious statement is that $S$ is a chain whenever $T$ is unbounded. To see this, let $u, u^{\prime} \in S$ have length $n$. Pick $k$ and $k^{\prime}$ with $P(u, k)$ and $P\left(u^{\prime}, k^{\prime}\right)$. We may assume that $k \geqslant k^{\prime}$. Since $T$ is unbounded, there is $v \in T$ such that $|v|=n+k$. Now $u \leqslant v$ and $u^{\prime} \leqslant v$ by our choice of $k$, and thus $u=u^{\prime}$.

Helmut Schwichtenberg ${ }^{3}$ has made the observation that if $u \in S$ and $T$ is unbounded, then

$$
T_{u}=\{v: u v \in T\}
$$

is unbounded; this is the subtree of $T$ with root $u$. Hence when one is after an infinite path in $T$, then it makes sense to consider $S$. Under suitable conditions $S$ will be the only infinite path in $T$.

By an infinite path $R$ in a tree $T$ we mean a linear spread (or, equivalently, an infinite chain-see above) such that $R \subseteq T$. Hence an infinite path in $A^{*}$ is nothing but a formal point of the formal topology on $A^{*}$ [26]. Notice that $S$ is an infinite path in $T$ if and only if $S \cap T$ is unbounded.

By an infinite path in a tree $T$ one usually understands an infinite sequence $\alpha$ of elements of $A$ (i.e. a mapping from $\mathbb{N}$ to $A$ ) whose finite initial segments

$$
\bar{\alpha} n=(\alpha(0), \ldots, \alpha(n-1))
$$

all belong to $T$. Every $\alpha$ of this sort gives clearly rise to the infinite path $\{\bar{\alpha} n: n \geqslant 0\}$ in the sense of this paper. Conversely, can one extract an infinite sequence $\alpha$ from an infinite path $R$ ? If $R$ is detachable and e.g. $A \subseteq \mathbb{N}$, then one can define $\alpha$ recursively by setting

$$
\alpha(n)=\min \{i \in\{0,1\}:(\bar{\alpha} n) i \in R\} .
$$

In general, however, one might need a suitable form of countable unique choice to pick, simultaneously for every $n$, the unique $u \in R$ of length $n$, and to gather them into a sequence.

Following [15] we say that a tree $T$ has uniformly at most one infinite path if

(1) $\forall n \exists m \forall v, w(|v|=n+m \wedge|w|=n+m \rightarrow v \in T \wedge w \in T \rightarrow \bar{v} n=\bar{w} n)$.

\footnotetext{
${ }^{3}$ Personal communication to the second author, JAIST, March 2010.
} 
If for any $n$ there is $m$ as in (1), then this $m$ can be taken as large as one pleases. Although no infinite sequence occurs in (1), it is equivalent to the definition one might expect [15]; see also [30]. Correspondents of (1) occur naturally in mathematical analysis, e.g. in the contexts of best approximations and of fixed points; see the references given in Footnote 1 above.

Lemma 2 Let $T$ be a tree with uniformly at most one infinite path. If $T$ is unbounded, then $S$ is a spread.

Proof By virtue of Lemma 1, part 1 it remains to be seen that if $u \in S$, then $u i \in S$ for some $i \in A$. Assume that $u \in S$, and take $k \geqslant 1$ with $P(u, k)$. For this $k$ and $n=|u|+1$, take $m \geqslant k$ as in (1). Note that $|u|<|u|+k<n+m$. Now there is $v \in T$ such that $|v|=n+m$. For any such $v$ we have $\bar{v}(|u|+k) \in T$ and thus $u \leqslant \bar{v}(|u|+k)$ by our choice of $k$. Hence $u i \leqslant v$ for some $i \in A$, for which $P(u i, m)$, and thus $u i \in S$. In fact, if $w \in T$ such that $|w|=n+m$, then $\bar{w} n=\bar{v} n=u i$ by our choice of $m$.

If $T$ is detachable and $A$ is finite, then Lemma 2 remains valid even if $T$ fails to be unbounded, in which sense $S$ is a generalisation of a longest path in $T$ [15]. In fact, if $u, k$, and $m$ are as in the foregoing proof, then (since $T$ is detachable and $A$ is finite) one can decide whether there is $v \in T$ such that $|v|=n+m$. If this is the case, then one can proceed as in the proof. Otherwise $T$ is bounded; whence $S=A^{*}$ by Lemma 1 , part 2 and thus $u i \in S$ for all $i \in A$.

We say that $R \subseteq A^{*}$ is uniformly absorbing (in $T$ ) if

$$
\forall n \exists m \forall w(|w|=n+m \rightarrow w \in T \rightarrow \bar{w} n \in R) .
$$

This notion corresponds to the one of a strong minimum [2]. Any two uniformly absorbing infinite paths in $T$ are equal. More precisely, if $R$ is an infinite path in $T$, and $R^{\prime}$ is uniformly absorbing in $T$, then $R \subseteq R^{\prime}$.

Lemma 3 Each of the following assertions implies the next:

(1) There is a chain $R$ which is uniformly absorbing in $T$.

(2) Thas uniformly at most one infinite path.

(3) Every unbounded tree $R \subseteq T$ is uniformly absorbing.

Lemma 4 If $S$ is unbounded, then it is uniformly absorbing in $T$. 
Proof For a given $n$ there is $u \in S$ with $|u|=n$, for which there is $m$ with $P(u, m)$. Any such $m$ is as required: if $w \in T$ has length $n+m$, then $u \leqslant w$ by our choice of $m$; whence $\bar{w} n=u$ and thus $\bar{w} n \in S$.

Theorem 5 The following statements are equivalent:

(1) $T$ is unbounded and has uniformly at most one infinite path.

(2) $S$ is an infinite path in $T$.

(3) There is an infinite path in $T$ which is uniformly absorbing.

Proof By Lemma 1, part 3 and Lemma 2 the first item implies the second, which in view of Lemma 4 implies the third item. According to Lemma 3 the first item follows from the third.

Under any of the equivalent conditions from Theorem $5, S$ is the one and only infinite path in $T$, and uniformly absorbing.

One might be tempted to enforce $S \subseteq T$ from the outset, by substituting $S \cap T$ for $S$. If one did so, then Lemma 1 would remain valid mutatis mutandis, whereas the proof of Lemma 2 would only work whenever $T$ is unbounded, in which case $S \subseteq T$ anyhow (Lemma 1, part 3).

Acknowledgements The second author wishes to thank Peter Aczel, Josef Berger, Milly Maietti, Giovanni Sambin, and Helmut Schwichtenberg for discussions and hints.

\section{References}

[1] Bauer, A., and P. Taylor, The Dedekind reals in abstract Stone duality. Math. Structures Comput. Sci. 19 (2009), 757-838; doi:10.1017/S0960129509007695.

[2] Berger, J., D. Bridges, and P. Schuster, The fan theorem and unique existence of maxima. J. Symbolic Logic 71 (2006), 713-720; doi:10.2178/js1/1146620167.

[3] Berger, J., and H. Ishihara, Brouwer's fan theorem and unique existence in constructive analysis. Math. Log. Quart. 51 (2005), 369-373; doi:10.1002/malq.200410038.

[4] Berger, J., H. Ishihara, and P. Schuster, The Weak König Lemma, Brouwer's Fan Theorem, De Morgan's Law, and Dependent Choice. 2009.

[5] Bishop, E., and D. Bridges, Constructive Analysis. Springer, Berlin etc., 1985. 
[6] Brattka, V., Borel complexity and computability of the Hahn-Banach theorem. Arch. Math. Logic 46 (2008), 547-564; doi:10.1007/s00153-007-0057-z.

[7] Bridges, D., On the foundations of best approximation theory. J. Approx. Theory 28 (1980), 273-292; doi:10.1016/0021-9045(80)90081-7.

[8] Bridges, D., A constructive proximinality property of finite-dimensional linear subspaces. Rocky Mountain J. Math. 11 (1981), 491-497.

[9] Bridges, D., Recent progress in constructive approximation theory. In: A.S. Troelstra, D. van Dalen, eds., The L.E.J. Brouwer Centenary Symposium. North-Holland, Amsterdam (1982), 41-50; doi:10.1016/S0049-237X(09)70121-1.

[10] Bridges, D.S., and F. Richman, Varieties of Constructive Mathematics. Cambridge University Press, 1987.

[11] Gelfond, M.G., On the relation between classical and constructive analysis. In: Studies in constructive mathematics and mathematical logic, Part V. Zap. Nauchn. Sem. Leningrad. Otdel. Mat. Inst. Steklov (LOMI) 32 (1972), 5-11.

[12] Hendtlass, M., and P. Schuster, Minima and best approximations in constructive analysis. J. Log. Anal. 3 (2011), Paper 5, 17 pp.; doi:10.4115/jla.2011.3.5.

[13] Ishihara, H., An omniscience principle, the König lemma and the HahnBanach theorem. Z. Math. Logik Grundlag. Math. 36 (1990), 237-240; doi:10.1002/malq.19900360307.

[14] Ishihara, H., Weak König's Lemma implies Brouwer's Fan Theorem: a direct proof. Notre Dame J. Formal Logic 47 (2006), 249-252; doi:10.1305/ndjfl/1153858649.

[15] Ishihara, H., Unique existence and computability in constructive reverse mathematics. In: S. B. Cooper, B. Löwe, A. Sorbi, eds., Computation and Logic in the Real World. Third Conference on Computability in Europe, CiE 2007. Siena, Italy, June 2007. Springer, Berlin and Heidelberg. Lect. Notes Comput. Sci. 4497 (2007) 368-377; doi:10.1007/978-3-540-73001-9_38.

[16] Ishihara, H., and P. Schuster, On the contrapositive of countable choice. Arch. Math. Logic 50 (2011) 137-143; doi:10.1007/s00153-010-0205-8.

[17] Kleene, S.C., Recursive functions and intuitionistic mathematics. In: L.M. Graves et al., eds., Proceedings of the International Congress of Mathematicians 1950. Amer. Math. Soc., Providence, R.I., 1952, 679-685.

[18] Kohlenbach, U., Effective moduli from ineffective uniqueness proofs. An unwinding of de La Vallée Poussin's proof for Chebycheff approximation. Ann. Pure Appl. Logic 64 (1993), 27-94; doi:10.1016/0168-0072(93)90213-W.

[19] Kohlenbach, U., New effective moduli of uniqueness and uniform a-priori estimates for constants of strong unicity by logical analysis of known proofs in best approximation theory. Numer. Funct. Anal. Optim. 14 (1993), 581-606; doi:10.1080/01630569308816541.

[20] Kohlenbach, U., and P. Oliva, Proof mining: a systematic way of analysing proofs in mathematics. Proc. Steklov Inst. Math. (2003), no. 3 (242), 136-164. 
Unique paths as formal points

[21] Kohlenbach, U., Applied Proof Theory: Proof Interpretations and their Use in Mathematics. Springer, Berlin and Heidelberg, 2008.

[22] Kren̆novič, V.Ja., Review of 'Constructive Functional Analysis'. MR0521982 (82k:03094).

[23] Lifshits, V.A., Investigation of constructive functions by the method of fillings. J. Soviet Math. 1 (1973), 41-47; doi:10.1007/BF01117468.

[24] Richman, F., The fundamental theorem of algebra: a constructive development without choice. Pacific J. Math.196 (2000), 213-230; doi:10.2140/pjm.2000.196.213.

[25] Richman, F., Spreads and choice in constructive mathematics. Indag. Math. (N.S.) 13 (2002), 259-267; doi:10.1016/S0019-3577(02)80009-X.

[26] Sambin, G., Intuitionistic formal spaces-a first communication. In: D. Skordev, ed., Mathematical Logic and its Applications, Conf. Proc. (Druzhba, Bulgaria, 1986). Plenum, New York and London (1987), 187-204.

[27] Sambin, G., Some points in formal topology. Theoret. Comput. Sci. 305 (2003), $347-$ 408; doi:10.1016/S0304-3975(02)00704-1.

[28] Schuster, P., Unique solutions. Math. Log. Quart. 52 (2006), 534-539; doi:10.1002/malq.200610012. Corrigendum: Math. Log. Quart. 53 (2007), 214.

[29] Schuster, P., Problems, solutions, and completions. J. Logic Algebr. Program. 79 (2010), 84-91; doi:10.1016/j.jlap.2009.02.007.

[30] Schwichtenberg, H., A direct proof of the equivalence between Brouwer's fan theorem and König's lemma with a uniqueness hypothesis. J. UCS 11 (2005), 2086-2095.

[31] Taylor, P., A lambda calculus for real analysis. J. Log. Anal. 2 (2010), Paper 5, 115 pp.; doi:10.4115/jla.2010.2.5.

[32] Weihrauch, K., Computability. Springer, Berlin, 1987.

[33] Weihrauch, K., Computable Analysis. An Introduction. Springer, Berlin, 2000; doi:10.1007/11494645_64.

Department of Computing Science, University of Göteborg

Sweden

Department of Pure Mathematics, University of Leeds

Leeds LS2 9JT, UK

coquand@chalmers.se, pschust@maths.leeds.ac.uk

http://www.cse.chalmers.se/ coquand/,

http://www. maths.leeds.ac.uk/ pschust/

Received: 9 August 2010 Revised: 1 July 2011

Journal of Logic \& Analysis 3:6 (2011) 